\title{
Using Feedback Technology Of Wideband MMIC Digital Attenuator
}

\author{
Chengpeng Liu ${ }^{1, a}$, Xiaojia Feng ${ }^{2, b}$ \\ ${ }^{1}$ Sichuan Institute of Solid State Circuits, China Electronics Technology Group Corp., \\ Chongqing 400060, P. R. China \\ ${ }^{2}$ Chongqing College of Electronic Engineering, Chongqing 401331, P. R. China \\ alcp54913@163.com, ${ }^{\mathrm{b}} 593653475 @ q q . c o m$
}

Keywords: Windband; Digital; Attenuator; Feedback technology; MMIC

\begin{abstract}
This paper mainly introduces the Wideband MMIC digital attenuator by using feedback technology. Firstly, the theories of basic GaAs digital attenuator MMIC's configurations are searched. Secondly, using feedback technology and normal configuration are contrasted, subsequently, appropriate topology is selected for each stage. This attenuator has been realized by $0.5 \mu \mathrm{m} \mathrm{GaAs}$ pHEMT process. Simulation results of the digital attenuator has $0.5 \mathrm{~dB}$ resolution and $31.5 \mathrm{~dB}$ dynamic attenuation range, over $\mathrm{DC} \sim 6 \mathrm{GHz}$, high attenuation accuracy is less than $\pm 0.7 \mathrm{~dB}$, Input Voltage Standing Wave Ratio(VSWR $\left.\mathrm{IN}_{\mathrm{N}}\right)$ was less than 1.2 and Output Voltage Standing Wave

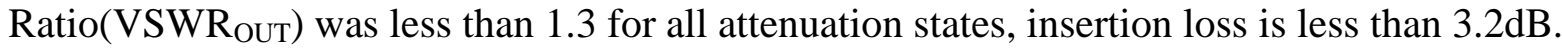

\section{Introduction}

Variable attenuators are required in many microwave and millimeter-wave systems for automatic gain control for receiver and transmitter systems, amplitude weighting for phased array radars and temperature compensation of microwave amplifiers. An attenuator specially offer better linearity, high power handling and easy and accurate control of attenuation over a large temperature range and MMIC process variations $^{[1 \sim 2]}$.

The attenuator has two types of control method, analog attenuator and digital attenuator. Digital attenuators offer better linearity, high power handling, and easy and accurate control of attenuation, So MMIC digital attenuators have gained lots of interest in recent years.

The requirements of the MMIC digital attenuators to be designed are as following: small size, high attenuation accuracy, low insertion phase shift, high reliability and low cost $\mathrm{t}^{[3 \sim 4]}$.

In this paper, we describes an Wideband MMIC digital attenuator, using feedback technology, The digital attenuator has obtained excellent performances and implements in 0.5um GaAs PHEMT MMIC.

\section{Circuit design}

Digital attenuation can be implemented by adopting several circuit topologies and different devices for switching. ublished literature on MMIC digital attenuators mainly rely on three basic types of topologies: i) Tee attenuator; ii) Bridged-Tee attenuator; iii)Pi attenuator. All of them relay on a signal through either a bypass line or an attenuation cell with RF switches. Fig.1, Fig.2 and Fig.3 show the topologies of Tee attenuator, Bridged-Tee attenuator and $\mathrm{Pi}$ attenuator. 


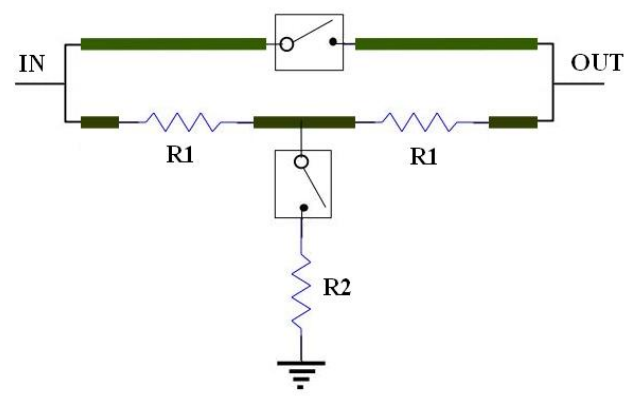

Figure. 1 Tee attenuator

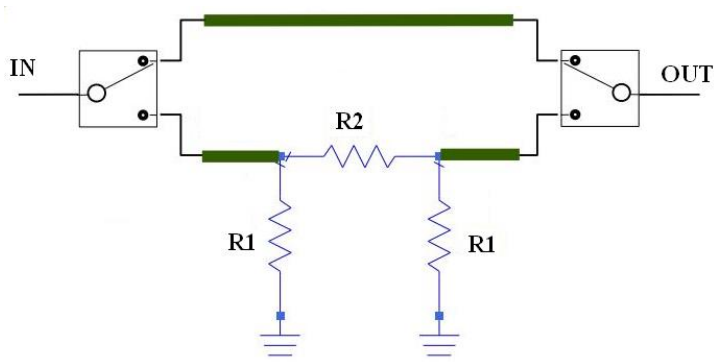

Figure. 3 Pi attenuator

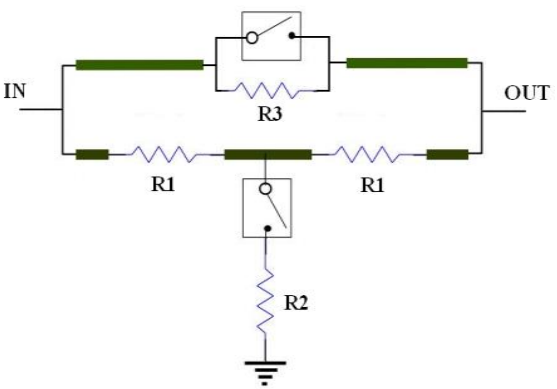

Figure. 2 Bridged-Tee attenuator

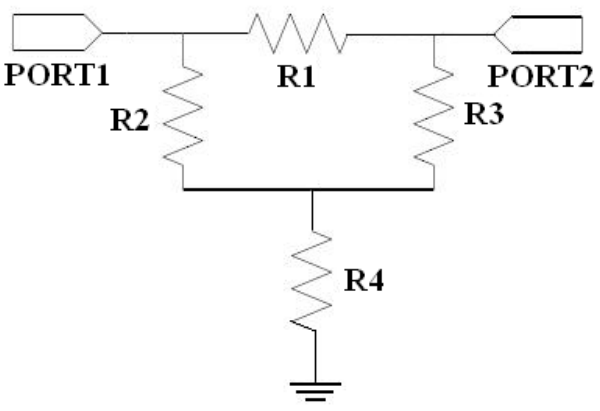

Figure. 4 Equivalent circuit of Bridged-Tee attenuator

In switched configurations, as shown in Fig.1, attenuation is obtained by alternating the active device gate voltages with two complementary values (typically PHEMT compatible $0 \mathrm{~V},-5 \mathrm{~V}$ ). The impedance level between drain and source of a cold-FET $\left(\mathrm{V}_{\mathrm{DS}}=0 \mathrm{~V}\right)$ is approximately equivalent to a small resistance $R_{\mathrm{ON}}$ (when $\mathrm{V}_{\mathrm{GS}}>\mathrm{V}_{\text {pinch-off }}$ )or to a small capacitance $\mathrm{C}_{\mathrm{OFF}}$ (when $\mathrm{V}_{\mathrm{GS}}<\mathrm{V}_{\text {pinch-off }}$ ). By analysing the circuits in Fig. 1, it is readily derived that a parasitic phase shift appears between the attenuator reference $\left(\mathrm{R}_{\mathrm{EF}}\right)$ and attenuating $(\mathrm{ON})$ states. Such unwanted effect is significant in phased arrays or similar systems in which the signal amplitude and phase must be precisely controlled. A partial solution, at system level, consists of over all phase and amplitude calibration; nevertheless, the single function deviations are to be kept as small as possible not to lose resolution. Such parasitic phase shift is due to the fact that $\mathrm{C}_{\mathrm{OFF}}$ is found alternately towards ground or between input and output ports in the switched attenuator states. As a result, the switched attenuator practically behaves as a low-pass filter in the $\mathrm{R}_{\mathrm{EF}}$ state and as a high-pass filter in the $\mathrm{ON}$ state, thus justifying the phase difference between the two states' transmission parameter $\mathrm{S}_{21}$. The solution proposed in this Letter consists in adding a properly sized reactive element to the circuit to compensate for the above-mentioned parasitic phase shift ${ }^{[5 \sim 7]}$.

To achieve good performance, the circuit configuration as well as the process conditions, should be selected properly.

The digital attenuator consists of switched PHEMTs, resistors, and microstrip lines. The switched PHEMTs in the circuit are controlled through $8 \mathrm{k} \Omega$ resistors of the gate poles, which provide enough radio frequency isolation between the gate of each switched PHEMT and the control sources. And the low value resistors are used to form the topologies for attenuators.

Bridged-Tee attenuator are used for the Wideband MMIC digital attenuator. Bridged-Tee attenuator (Fig.2) has good performance in insertion loss, $\mathrm{VSWR}_{\mathrm{IN}}$ and $\mathrm{VSWR}_{\mathrm{OUT}}$ also has better performance than Pi attenuator ${ }^{[8 \sim 11]}$.

Referring to Fig.4, The equivalent circuit of Bridged-Tee attenuator shows $\left(\mathrm{R}_{2}=\mathrm{R}_{3}\right)$ : 


$$
Z=\left[\begin{array}{ll}
\frac{R_{1} Z_{0}}{R_{1}+2 Z_{0}}+R_{4} & \frac{Z_{0}^{2}}{R_{1}+2 Z_{0}}+R_{4} \\
\frac{Z_{0}^{2}}{R_{1}+2 Z_{0}}+R_{4} & \frac{R_{1} Z_{0}+Z_{0}^{2}}{R_{1}+2 Z_{0}}+R_{4}
\end{array}\right]=\left[\begin{array}{ll}
Z_{11} & Z_{12} \\
Z_{21} & Z_{22}
\end{array}\right]
$$

The characteristic impedance of transfers lines connect to port 1 and port 2 are $Z_{\text {in }}$ and $Z_{\text {out }}$, so:

$$
Z=\left[\begin{array}{cc}
\frac{Z_{11}}{Z_{\text {in }}} & \frac{Z_{12}}{\sqrt{Z_{\text {in }}+Z_{\text {out }}}} \\
\frac{Z_{21}}{Z_{\text {in }} \cdot Z_{\text {out }}} & \frac{Z_{22}}{Z_{\text {out }}}
\end{array}\right]=\left[\begin{array}{cc}
Z_{11} & Z_{12} \\
Z_{21} & Z_{22}
\end{array}\right]
$$

The S-parameters are:

$$
\begin{aligned}
& S_{11}=\frac{|Z|-1+Z_{11}-Z_{22}}{|Z|+1+Z_{11}+Z_{22}} \\
& S_{22}=\frac{|Z|-1-Z_{11}+Z_{22}}{|Z|+1+Z_{11}+Z_{22}} \\
& S_{21}=\frac{2 Z_{21}}{|Z|+1+Z_{11}+Z_{22}} \\
& S_{12}=\frac{2 Z_{12}}{|Z|+1+Z_{11}+Z_{22}}
\end{aligned}
$$

And:

$$
\begin{aligned}
& L=20 \lg \left|S_{21}\right| \\
& 10 \lg \left|S_{11}\right|=-\infty
\end{aligned}
$$

From (1) (8), the value of $\mathrm{R}_{1}$ and $\mathrm{R}_{4}$ in Bridged-Tee attenuator can show below:

$$
\begin{aligned}
& R_{1}=Z_{0}\left(10^{\frac{L}{20}}-1\right) \\
& R_{4}=\frac{Z_{0}}{10^{\frac{L}{20}}-1}
\end{aligned}
$$

From (9), the value of $\mathrm{R}_{1}$ and $\mathrm{R}_{4}$ can be calculated by given attenuation bits.

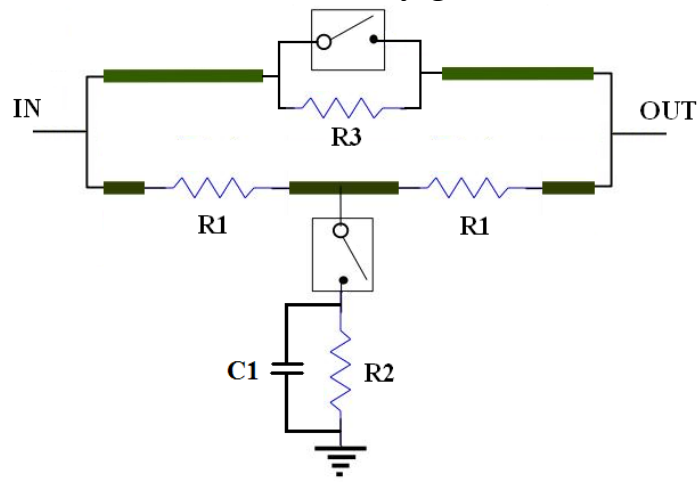

Figure. 5 Bridged-Tee attenuator with feedback capacitance

Referring to Fig.5, Bridged-Tee attenuator with feedback capacitance is shown. these aspects are achieved by providing a feedback capacitance $\mathrm{C} 1$, under the same conditions, the novel configuration can extended the band width. 


\section{Simulation results}

The MMIC Digital Attenuator have been presented based on the ADS2008. With feedback capacitance or not,there is an obvious gap between the performance of them:

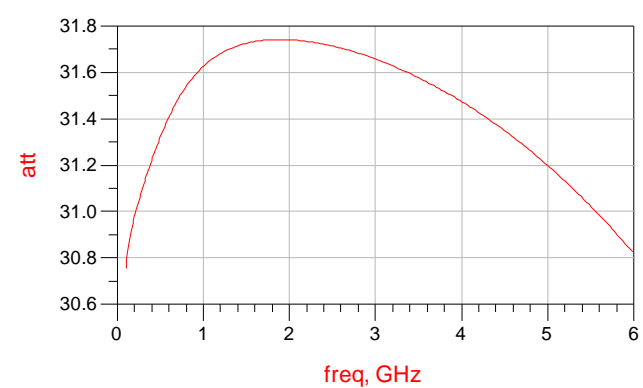

Figure. 6 31.5dBAttenuation Bit

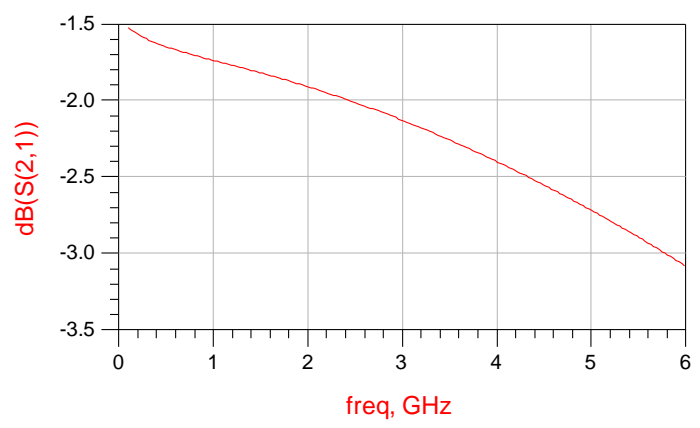

Figure. 8 Simulated Insertion Loss

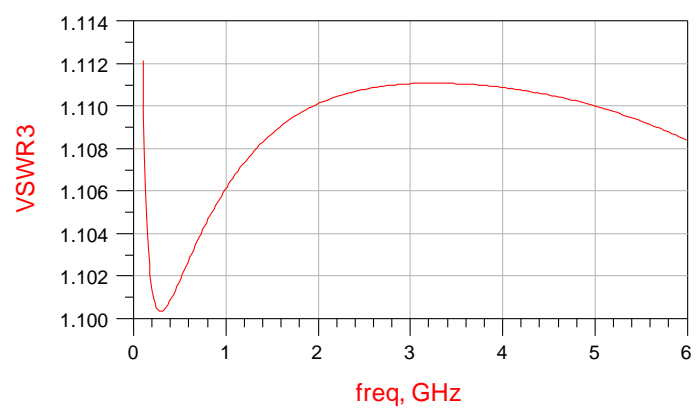

Figure. 10 Simulated Iutput Return Loss

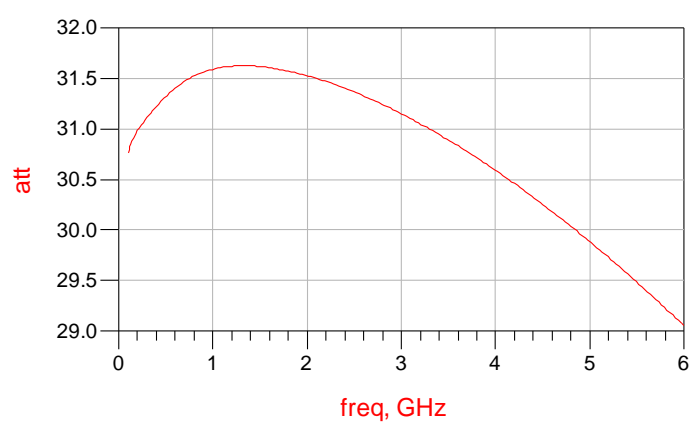

Figure. 7 31.5dBAttenuation Bit

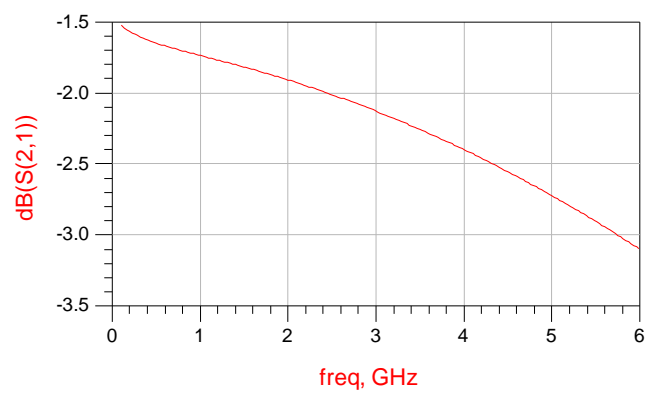

Figure. 9 Simulated Insertion Loss

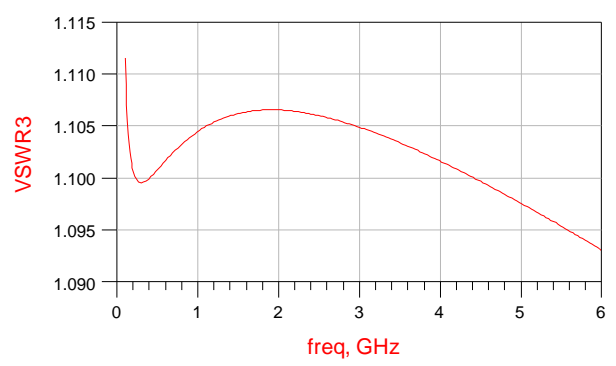

Figure. 11 Simulated Output Return Loss(on) 


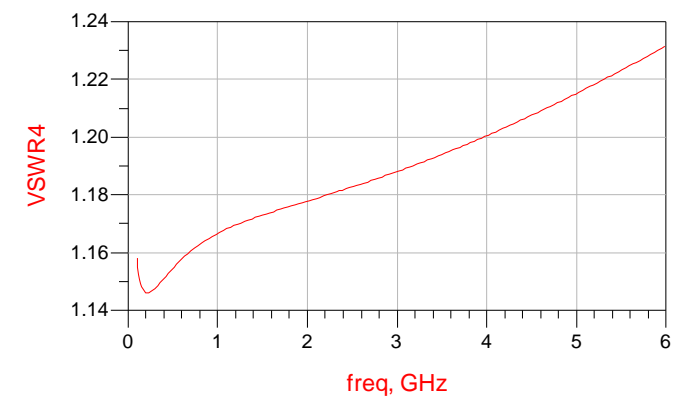

Figure. 12 Simulated Iutput Return Loss

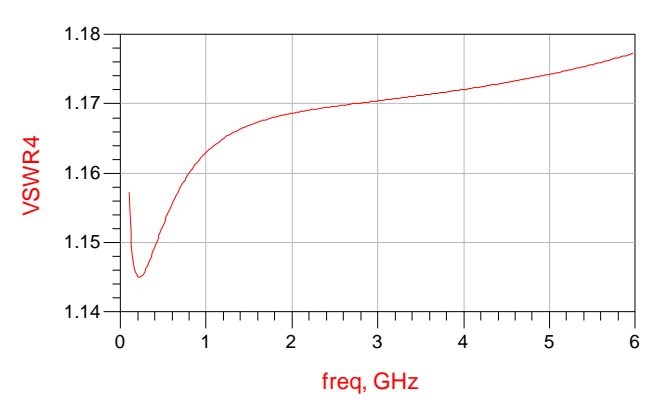

Figure. 13 Simulated Output Return Loss(on)

With feedback capacitance, over DC $6 \mathrm{GHz}$, insertion loss is lower than $3.2 \mathrm{~dB}$ (Fig.8), at $31.5 \mathrm{~dB}$ attenuation bit, the attenuation accuracy is less than $\pm 0.7 \mathrm{~dB}$ (Fig.6), The VSWR $\mathrm{IN}_{\mathrm{IN}}$ was always less than 1.12 (Fig.10) and the VSWR OUT was always less than 1.24(Fig.12).

Without feedback capacitance over DC $6 \mathrm{GHz}$, insertion loss is lower than $3.2 \mathrm{~dB}$ (Fig.9), at $31.5 \mathrm{~dB}$ attenuation bit, the attenuation accuracy is about $2.5 \mathrm{~dB}$ (Fig.7), The $\mathrm{VSWR}_{\mathrm{IN}}$ was always less than

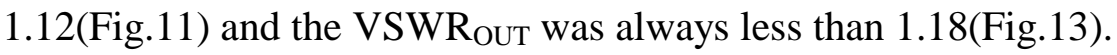

\section{Conclusion}

The theory of the Wideband MMIC digital attenuator with feedback technology are presented. appropriate topology is selected for stages. To ensure high yield, Performance redundancy optimization strategy is used in design. the simulation results of the developed MMIC show that providing a feedback capacitance, the digital attenuator could work at higher frequency.with the feedback capacitance the attenuation accuracy is less than $\pm 0.7 \mathrm{~dB}$ compare to about $2.5 \mathrm{~dB}$ without feedback capacitance.

This proposed MMIC has shown excellent performance covering DC $\sim 6 \mathrm{GHz}$ for digital attenuator.

\section{References}

[1] Cheng Peng Liu; Xin Xu: X-Band MMIC Digital Attenuator With Low Phase Shift [J]. 2014(6).

[2] Chengpeng Liu; Luncai Liu; Guoqiang Wang; Zhengrong He; Wei Zou: A novel ultra wideband low noise amplifier [C]. Proceedings of ICFCIT, 2013

[3] Yong-Sheng Dai; Yong-Sheng Dai; Xiao-Jim Chen; Jin-Ting Lin: A NOVEL DC-SOGHZ MMIC VARIABLE ATTENUATOR [C]. Proceedings of ICMMWTP, 2002: p167-170.

[4] W. Ciccognani, F. Giannini, E. Limiti and P.E. Longhi: Compensating for parasitic phase shift in microwave digitally controlled attenuators [J]. ELECTRONICS LETTERS, 2008(44):NO.12

[5] Yong-Sheng Dai, Jie Zhang, Bing-Qing Dai, Zhi-DongSong, Gui-Xiang Qian, Shao-Bo Chen, Wen-Kan Zhou.: An Ultra Broadband 2-18GHz 6-Bit PHEMT MMIC Digital Attenuator with Low Insertion Phase Shift [C]. Proceedings of 2010 IEEE International Conference on Ultra-Wideband, IEEE, 2010: p167-170

[6] Amir Effendy; Ashraff Al: The Design of $5 \mathrm{~dB}$ Attenuator in Coplanar Waveguide for DC to 67 GHz [C]. IEEE, RFM2011, 2011: p246-249.

[7] Baldwin, G.L. ; McCreary, J., "An MOS Digitally Controlled Analog Attenuator for Voiceband Signals," IEEE Trans. on Communications, vol. 27,no.2, pp.332-337,1979 
[8] Na Chen: A Millimeter-wave 6-bit GaAs Monolithic Digital Attenuator with Low Insertion Phase Shift [C]. Proceedings of International Workshop on Microwave and Millimeter Wave Circuits and System Technology, IEEE, 2013:p440-443.

[9] Granger-Jones, M.; Nelson, B.; Franzwa, E., "A broadband high-dynamic-range voltage-controlled attenuator MMIC with an IIP3 > +47 dBm over entire 30-dB analog control range," in Proc. Microwave Symposium Digest, , 2011, pp. 1.

[10] Xing Wen ; Fa-Xin Yu ; Ling-Ling Sun, “A Novel Ultra Broadband 8-45 GHz 4-Bit GaAs Pseudomorphic High Electron Mobility Transistors (pHEMT) Monolithic Digital Attenuator Used for Gain Control of Transceivers," in Proc. Innovative Computing, Information and Control, 2009, pp. 519-522.

[11] Gupta, R.K. ; Reynolds, J.H. ; McNally, P.J., "Modeling and CAD of an Ultra-Broadband Monolithic 5-bit Digital Attenuator," in Proc. Microwave Conference, 1988, pp.151-155. 